\title{
Laminar Mixing in Diluted and Undiluted Fuel Jets Upstream from Lifted Flames
}

\author{
A. REVUELTA, A. L. SÁNCHEZ* \\ Area de Mecánica de Fluidos, Departamento de Ingeniería Mecánica, Universidad Carlos III de Madrid, 28911 \\ Leganés, Spain
}

and

\author{
A. LIÑ̃́N \\ Departamento de Motopropulsión y Termofluidodinámica, E. T. S. I. Aeronáuticos, Universidad Politécnica de \\ Madrid, 28040 Madrid, Spain
}

\begin{abstract}
The boundary-layer approximation is used to describe the frozen mixing process taking place when a fuel jet of radius $a$ discharges into stagnant air. The results are applicable to the calculation of lifted flames stabilized in round laminar jets with relatively large Reynolds numbers, $R e$, for which the proposed formulation provides a detailed description for the velocity and composition fields encountered by the propagating triple flame formed at the base of the lifted flame. The problem is integrated for relevant values of the flow parameters, including values of the stoichiometric air-to-fuel mass ratio $S$ of order unity, when the lifted flame is located in the region of jet development, corresponding to distances from the injector of order Re $a$. Further attention is given to the relevant case $S \gg 1$, corresponding to typical conditions of undilute hydrocarbon-air flames, for which the resulting lifted flames are stabilized at relatively large distances from the injector, of order $S R e a$. It is seen that Schlichting asymptotic solution, which corresponds to a point source of momentum, is then applicable to describe the mixing process upstream from the lifted flame. Improved accuracy is sought by introducing expansions for the velocity components and for the reactant mass fractions in powers of $S^{-1}$. The resulting development shows in particular that the first-order correction to the leading-order solution is equivalent to the introduction of a virtual origin for the axial coordinate. It is shown that the magnitude of the required translation, which is equal for the velocity and composition fields, must be determined from continuity considerations. As an illustrative example, the resulting description is used to calculate flame fronts with $S \gg$ 1 in the thermal-diffusive approximation.
\end{abstract}

\section{INTRODUCTION}

Extensive experimental and analytical work aimed at clarifying the stabilization mechanism of laminar lifted flames in fuel injectors has been developed in recent years by Chung and co-workers (see $[1,2]$ and the more recent work cited in [3]). The base of the lifted flame is seen to be a triple flame that propagates into the partially premixed stream that forms upstream as a result of the frozen mixing process between the fuel jet and the surrounding air. For the large values of the Reynolds number typical of most practical applications, the flame front is thin compared with the jet radius, and its local structure is that of a planar premixed front, whose propagation velocity is known to be maximum where the mixture is nearly stoichiometric. Correspondingly, the premixed front

\footnotetext{
* Corresponding author: E-mail: asanchez@ing.uc3m.es
}

moves faster along the stoichiometric surface $\Sigma_{s}$ and exhibits a characteristic structure with a lean branch and a rich branch. On the lean side, the premixed flame consumes all the available fuel, leaving behind oxygen that reacts in a trailing diffusion flame with the fuel left behind by the rich branch. Because of their reduced propagation velocity, the lean and rich branches of the flame front curve backwards from the leading stoichiometric line.

For the triple flame to remain lifted, its propagation velocity $U_{f}$ must be approximately equal to the local flow velocity at the stoichiometric surface. The computation of the frozen mixing process upstream from the lifted flame provides the velocity along the stoichiometric surface, which can then be used to calculate the lift-off height by equating the resulting value to the propagation velocity of the triple flame, a procedure proposed by Chung [1-3]. Because of curvature and thermal expansion effects [4-6], 
this propagation velocity, $U_{f}$, differs from the propagation velocity of a stoichiometric premixed flame, $S_{L}$ although it remains of the same order of magnitude. As a result, although the computation of lifted flames incorporating the simplifying assumption $U_{f}=S_{L}$ is seen to yield results in reasonable agreement with measurements [2], improved accuracy is obtained when a more careful evaluation of $U_{f}$ is incorporated [7].

If the Reynolds number of the jet $R e=U_{o} a / \nu$, constructed from the characteristic jet velocity, $U_{o}$, injector radius, $a$, and kinematic viscosity, $v$, is sufficiently larger than unity, and yet sufficiently small for the resulting steady laminar jet to remain stable, then the composition and velocity fields upstream from the lifted flame can be determined by integrating the boundarylayer form of the conservation equations. Measuring the axial coordinate with $R e a$ and the radial coordinate with $a$ enables the Reynolds number to be scaled out of the boundary-layer formulation, yielding the parabolic problem given below in Eqs. 1-6. Although approximate descriptions of the region of jet development, corresponding to distances of order $\operatorname{Re} a$, are available $[8,9]$, numerical integration is necessary for an accurate calculation of the mixing process in this region. Sufficiently far from the injector, that is, at distances much larger than $\operatorname{Re} a$, the resulting problem is seen to admit a selfsimilar description, originally derived by Schlichting [10] and Squire [11], in which explicit analytic expressions are available for the composition and velocity fields. As explained below, use of this approximation to describe frozen mixing upstream from the triple flame is only appropriate when, as typically occurs in undiluted hydrocarbon-air flames, the stoichiometric air-to-fuel mass ratio $S$ (the amount of air that one needs to mix with the unit mass of gas of the fuel stream to generate a stoichiometric mixture) is large, a case for which the stoichiometric surface $\Sigma_{s}$ is seen to extend over distances of order $S \operatorname{Re} a \gg R e a$.

The conditions for the applicability of the boundary-layer approximation and its associated far-field Schlichting solution are not clearly stated by many authors working on lift-off flames. For instance, in their interesting work, Chen and Bilger [7] identified the boundary- layer approximation with the selfsimilar Schlichting solution, and claimed that the full NavierStokes equations need to be integrated to describe laminar mixing as the jet develops, that is, at distances from the injector of order Re $a$. As a result of the use of the Navier-Stokes equations, the Reynolds number remains as a parameter in their formulation, an unnecessary complication that could have been avoided should the authors have used the boundarylayer formulation given below.

The recent paper by Ghosal and Vervish [12] makes use of Schlichting solution to calculate the flow velocity along $\Sigma_{s}$. Their work is an extension of Chung's procedure [1-3] to calculate lift-off heights, with the assumption $U_{f}=S_{L}$ being replaced with an approximate expression for $U_{f}$ valid only for $S=1$, which incorporates thermal expansion as well as curvature effects. By equating this expression to the flow velocity obtained for $S=1$ along $\Sigma_{s}$ the authors derived an implicit equation that was employed to predict lift-off and blow-out behaviors. Although Schlichting solution is not applicable in the case studied $(S=1)$, for which $\Sigma_{s}$ extends downstream only to distances of order $\operatorname{Re} a$, their simplified analysis provides qualitative understanding of the complex processes involved.

Our analysis of the far-field solution will address in particular the concept of the virtual origin arising in Schlichting solution. This translation of the axial coordinate is often introduced as an ad-hoc remedy for the singular behavior that appears at the origin in the asymptotic description for the axial velocity. We shall see that, in reality, the virtual origin arises naturally when seeking a first-order correction to the Schlichting asymptotic solution. This development, which is seen to be necessary for increased accuracy, employes the reciprocal of the distance to the injector as an asymptotically small quantity. We shall show that the value of the translation associated with the virtual origin cannot be arbitrarily selected; its value, which turns out to be equal for the velocity field and for the composition field, must be computed from continuity considerations by integrating numerically the boundary-layer equations in the region of jet development. Far-field mixing descriptions including virtual origins have been 
used previously to improve accuracy in calculations of lift-off heights [13, 14].

Our paper is therefore intended to contribute clarification to the above issues, one of the objectives being that of stating clearly the limitations on the use of Schlichting solution to describe frozen mixing in the round jet. The boundary-layer problem will be formulated, and the resulting equations will be integrated for characteristic values of the relevant parameters. The far-field solution, applicable only for large values of $S$, will be developed for reactant diffusivities that differ from the viscous diffusivity. Further comments on the stabilization of lift-off flames in round fuel jets are offered in the final section, which also includes the constant-density computation of flame fronts in the developed region corresponding to distances from the injector of order $S \operatorname{Re} a$.

\section{THE BOUNDARY-LAYER FORMULATION}

Mixing can be characterized in terms of the spatial variation of the local equivalence ratio $\phi=S \hat{Y}_{F} / \hat{Y}_{O}$, where $\hat{Y}_{F}$ and $\hat{Y}_{O}$ are the mass fractions of fuel and oxygen scaled with their corresponding initial values $Y_{F O}$ and $Y_{O A A}$, and $S=s Y_{F O} / Y_{O A}$ is the stoichiometric mass ratio, with $\mathrm{s}$ denoting the mass of oxygen needed to burn the unit mass of fuel. The condition $\phi=1$ defines the location of the stoichiometric surface, $\Sigma_{s}$ which separates the inner region where the mixture is rich $(\phi>1)$ from the outer region where the mixture is lean $(\phi<1)$.

For simplicity in the description, the mean molecular weight is assumed to be constant and the temperatures of the fuel and of the surrounding air are assumed to be equal to $T_{o}$ thereby yielding a flow field with constant density, $\rho$, and constant transport properties when evaluating the frozen distributions of velocity and reactants. The resulting flow can be determined in the boundary-layer approximation corresponding to $R e \gg 1$ by solving numerically the equations

$$
\begin{aligned}
& \frac{\partial u}{\partial x}+\frac{1}{r} \frac{\partial(r v)}{\partial r}=0 \\
& u \frac{\partial u}{\partial x}+v \frac{\partial u}{\partial r}=\frac{1}{r} \frac{\partial}{\partial r}\left(r \frac{\partial u}{\partial r}\right)
\end{aligned}
$$

$$
\begin{aligned}
& u \frac{\partial \hat{Y}_{F}}{\partial x}+v \frac{\partial \hat{Y}_{F}}{\partial r}=\frac{1}{S_{F}} \frac{1}{r} \frac{\partial}{\partial r}\left(r \frac{\partial \hat{Y}_{F}}{\partial r}\right) \\
& u \frac{\partial \hat{Y}_{o}}{\partial x}+v \frac{\partial \hat{Y}_{o}}{\partial r}=\frac{1}{S_{o}} \frac{1}{r} \frac{\partial}{\partial r}\left(r \frac{\partial \hat{Y}_{o}}{\partial r}\right)
\end{aligned}
$$

with initial conditions

$x=0$,

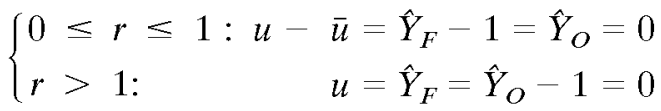

and with boundary conditions

$$
\begin{aligned}
& r=0: \frac{\partial u}{\partial r}=v=\frac{\partial \hat{Y}_{F}}{\partial r}=\frac{\partial \hat{Y}_{o}}{\partial r}=0 ; \\
& r \rightarrow \infty: u=\hat{Y}_{F}=\hat{Y}_{O}-1=0 .
\end{aligned}
$$

The boundary condition for the velocity as $r \rightarrow$ $\infty$ corresponds to a fuel jet discharging into stagnant air. The terms involving axial molecular transport of momentum and species, which are a factor $R e^{-2}$ smaller than those retained in Equations 2-4, have been left out of the equations in this approximation. The spatial coordinates and the velocity components are scaled with their characteristic values in the region of jet development. Thus, $x$ and $r$ are the axial and radial coordinates scaled with $\operatorname{Re} a$ and $a$, respectively, and the axial and radial velocity components $u$ and $v$ are scaled with $U_{\mathrm{O}}=$ $\left[J /\left(\rho \pi a^{2}\right)\right]^{1 / 2}$ and $v / a$, with $J$ denoting here the momentum flux of the fuel jet. The initial velocity distribution $\bar{u}(r)$ at $x=0$ depends on the shape of the jet velocity profile. Cases of interest include that of a uniform profile $\bar{u}=1$ and that of a fully developed Poiseuille profile $\bar{u}$ $=\sqrt{3}\left(1-r^{2}\right)$. The transport Schmidt numbers of fuel and oxidizer, $S_{F}$ and $S_{O}$, are the ratio of the kinematic viscosity to the molecular diffusivity of the corresponding reactant. Radial integration of Equations 2-4 yields the integral conservation laws for momentum and reactants

$$
\begin{aligned}
\int_{0}^{\infty} 2 r u^{2} d r & =\int_{0}^{\infty} 2 r u \hat{Y}_{F} d r / g_{o} \\
& =\int_{0}^{\infty} 2 r u\left(1-\hat{Y}_{o}\right) d r / g_{o}=1 .
\end{aligned}
$$




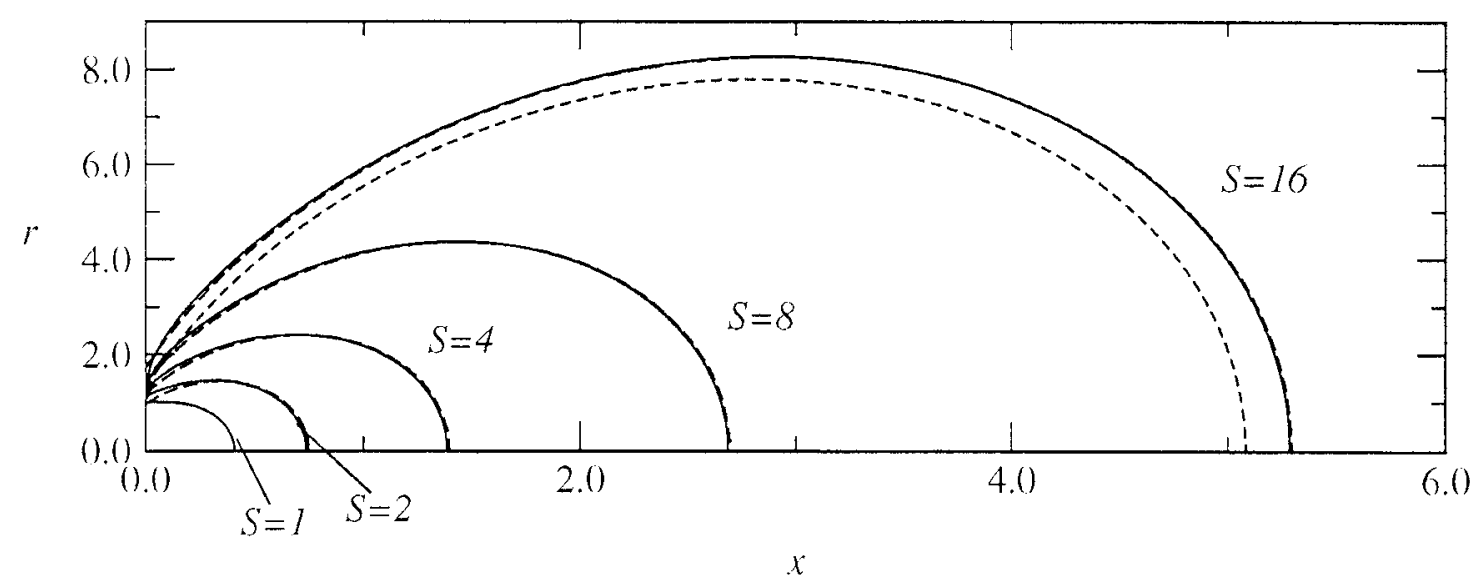

Figure 1. The stoichiometric surfaces $\phi=1$ obtained for different values of $S$ from integration of $1-6$ with $S_{F}=1,0, S_{o}=$ 0.73 , and with a Poiseuille profile $\bar{u}=\sqrt{3}\left(1-\mathrm{r}^{2}\right)$; the long dashed line corresponds to the two-term Schlichting prediction 27 and the dashed line corresponds to the leading-order result 26.

which are satisfied by the solution of (1)-(4). In the formulation, the constant

$g_{o}=\int_{0}^{1} 2 r \bar{u} d r$

is proportional to the initial volume flux, becoming $g_{o}=1$ for the initially uniform velocity profile and $g_{o}=\sqrt{3} / 2$ for Poiseusille flow. Integration of 1 gives

$\int_{0}^{\infty} 2 r u d r=g_{0}+2 \int_{0}^{x} \chi\left(x^{\prime}\right) d x^{\prime}$

for the jet volume flux at a given $x$ location, revealing that the volume flux driven by the developing jet increases from the initial value $g_{o}$, because of outer fluid that is being entrained. The entrainment rate $\chi(x)=-(r v)_{r \rightarrow \infty}$ must be determined from the solution to 1 and 2 , giving a distribution, which is exhibited for instance in [15] for both uniform and Poiseuille initial profiles, that decays for increasing values of $\mathrm{x}$ from the initial values $\chi=1.269 x^{-1 / 3}$ (Poiseuille) and $\chi=0.619 x^{-1 / 2}$ (uniform) for $x \ll 1$ towards the constant value $\chi=4$ for $x \gg 1$.

Integration of Equations. 1-6 determines the frozen mixing process upstream from the lifted flame. The results shown below in Figs. 1-4 are obtained with a fully developed Poiseuille profile, a configuration often encountered in the experiments reported in the literature [1-3]. In all cases computed here, the oxidizer Schmidt number is assumed to be $S_{0}=0.73$, as corresponds approximately to the normal diffusivity of air.

The condition $\phi=1$ determines the location of the stoichiometric surface $\Sigma_{s}$, which is shown in Fig. 1 for $S_{F}=1.0$ and for different values of $S$. A related quantity of practical interest is the flow velocity $u=u_{s}$ along $\Sigma_{s}$. Axial distributions of $u_{s}$ obtained for $S=16$ and $S=1$ with three different characteristic values of $S_{F}$, are exhibited in Figs. 2 and 3; dashed curves in Figs. 1-3, corresponding to the far-field solution, are to be discussed later.

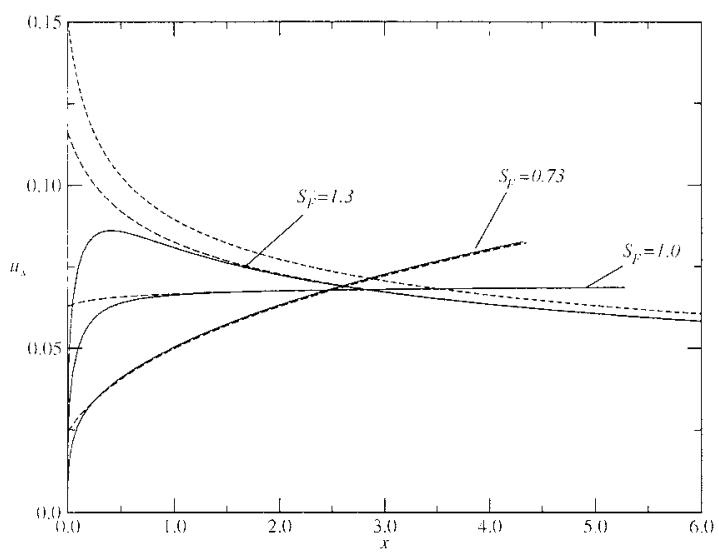

Figure 2. The axial variation of $u_{s}$ for $S_{o}=0.73$ and $S=16$ as obtained from integration of Eqs. 1-6 (solid lines), and from evaluation of $\mathrm{Eq} .28$ (dashed lines) and Eq. 29 (long dashed lines). 


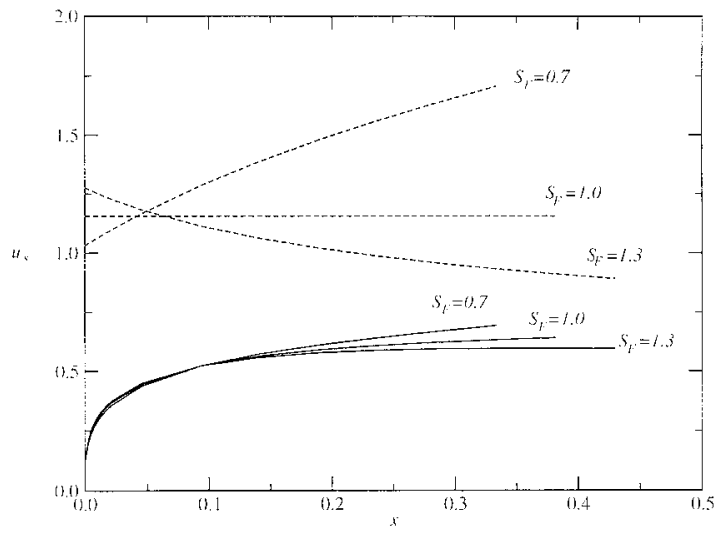

Figure 3. The axial variation of $u_{s}$ for $S_{o}=0.73$ and $S=1$ as obtained from integration of Eqs. 1-6 (solid lines) and from evaluation of Eq. 28 (dashed lines).

According to Chung's procedure, the value of $u_{s}$ must be equated to the propagation velocity of the triple flame to determine the lift-off height. For the resulting solution to be stable, $u_{s}$ must be a decreasing function of $x$ at the lift-off location. On the other hand, if $u_{s}$ is an increasing function of $x$ at the lift-off location, then the value of $x$ determined from the condition $u_{s}=$ $U_{f} U_{o}$ corresponds to the farthermost location where, by applying an ignition source, one may generate an ignition front that propagates upstream to the injector rim. Because a Poiseuille profile is assumed at the jet exit in Figs. 2 and 3, the value of $u_{s}$ starts increasing in all cases from the zero value at the injector rim, thereby precluding the stabilization of lifted flames in the vicinity of the injector (aside from the cases when the diffusion flame is attached to the near wake of the injector). With the flow velocity at the injector wall being necessarily zero, this characteristic of the $u_{s}$ distribution is necessarily present in other configurations regardless of detailed shape of the initial velocity distribution, except for the idealized case of a uniform velocity profile $\bar{u}=1$ with a vanishing boundarylayer thickness at $r=1$, for which the value of $u_{s}$ is seen to approach a finite value as $x \rightarrow 0$.

In Schlichting solution $[10,11]$, the leadingorder evolution of $u_{s}$ with axial distance, given below in Eq. 28, is seen to be dependent on the sign of $\left(S_{F}-1\right)$ [2]. Thus, for $S_{F}>1$, the value of $u_{s}$ decreases with distance, while $u_{s}$ remains constant when $S_{F}=1$ and increases with distance when $S_{F}<1$. Analyses based on Schlicht- ing description thereby yield $S_{F}>1$ as a requirement for flame stabilization. As seen in Fig. 2, this criterion applies for $S \gg 1$, when the stoichiometric surface extends downstream far from the injector, to a far-field region where Schlichting description becomes accurate, and where the sign of $\left(S_{F}-1\right)$ determines whether $u_{s}$ decays with distance for $x \gg 1$.

For $S$ of order unity, on the other hand, the stoichiometric surface develops over distances of order Re $a$ from the injector (values of $x$ of order unity). In this developing region, the distribution of $u_{s}$ is very sensitive to the initial velocity profile. For instance, for the Poiseuille profile used in Fig. 3 the value of $u_{s}$ is seen to increase monotonically for the three values of $S_{F}$ considered. Clearly, the stability criterion $S_{F}$ $>1$ developed from the Schlichting approximation would not be applicable in this case. In general, in configurations with $S$ of order unity the integration of $1-6$ is necessary to compute the value of $u_{s}$ and determine whether a lifted flame may exist.

\section{FIRST-ORDER CORRECTION TO THE ASYMPTOTIC SCHLICHTING SOLUTION}

As previously mentioned, the solution to 1-6 can be simplified when $S \gg 1$, a case for which $\Sigma_{S}$ is seen to lie far in the oxidizer domain. Two different regions can be identified in the solution: one corresponding to axial distances from the jet exit of order $\operatorname{Re} a$, and the second corresponding to the developed region emerging farther downstream, which extends to distances of order $S$ Re $a$.

The stoichiometric surface is initially located on the oxidizer side of the thin annular mixing layer that develops from the injector rim. The mixing layer continuously grows downstream, so that at distances from the jet of order $R e a$ (values of $x$ of order unity) transverse mixing acts across the fuel jet and starts to reduce significantly the value of $\hat{Y}_{F}$ from its initial unity value. Because $\Sigma_{S}$ is located where $\hat{Y}_{F} \sim S^{-1}$, the stoichiometric surface remains on the air side of the jet, at a radial distance of order $a$ (corresponding to values of $r$ of order unity). The structure of this region of initial development can only be described numerically solving 
the whole problem 1-6. The jet radius continues to grow downstream to values of order $S a$ $(r \sim S)$ at a distance of order $S \operatorname{Re} a(x \sim S)$, where $\hat{Y}_{F},\left(1-\hat{Y}_{o}\right)$, and $u$ are all of order $S^{-1}$. This second region, corresponding to values of $x$ in the range $1 \ll x \lesssim S$, admits an asymptotic description in which the jet acts in the first approximation as a point source of momentum [10]. As seen below, the initial mass flux of the jet must be accounted for in the first-order correction to the asymptotic solution.

For $S \gg 1$ the stoichiometric surface extends axially to rescaled distances $x \sim S$, where the jet radius increases linearly with distance. The solution for Equations. 1-4 in this region of $x \sim S$ $\gg 1$ can be sought by introducing a selfsimilar coordinate $\zeta=r / x$. The description of the velocity field requires introduction of an expansion in powers of $x$ for the stream function

$\psi=x F_{0}(\zeta)+F_{1}(\zeta)+O\left(x^{-1}\right)$

defined such that $u=x^{-1} F_{0}^{\prime} / \zeta+x^{-2} F_{1}^{\prime} / \zeta+$ $O\left(x^{-3}\right)$ and $r v=-F_{0}+\zeta F_{0}^{\prime}+x^{-1} \zeta F_{1}^{\prime}+$ $O\left(x^{-2}\right)$, where the prime ' denotes differentiation with respect to $\zeta$. Introducing these alternative variables into Eqs. 1, 2, 6, and 7 yields the problems

$$
\begin{gathered}
F_{0}^{\prime \prime \prime}+F_{0}^{\prime \prime}\left(F_{0}-1\right) / \zeta+F_{0}^{\prime}\left(1-F_{0}+\zeta F_{0}^{\prime}\right) / \zeta^{2}=0 \\
\left\{\begin{array}{l}
F_{0}(0)=\left(F_{0}^{\prime} / \zeta\right)^{\prime}(0)=F_{0}^{\prime}(\infty)=0 \\
2 \int_{0}^{\infty} F_{0}^{\prime 2} / \zeta \mathrm{d} \zeta=1
\end{array}\right.
\end{gathered}
$$

and

$$
\begin{gathered}
F_{1}^{\prime \prime \prime}+F_{1}^{\prime \prime}\left(F_{0}-1\right) / \zeta+F_{1}^{\prime}\left(1-F_{0}+3 \zeta F_{0}^{\prime}\right) / \zeta^{2}=0 \\
\left\{\begin{array}{l}
F_{1}(0)=\left(F_{1}^{\prime} \zeta\right)^{\prime}(0)=F_{1}^{\prime}(\infty)=0 \\
\int_{0}^{\infty} F_{0}^{\prime} F_{1}^{\prime} / \zeta \mathrm{d} \zeta=0
\end{array}\right.
\end{gathered}
$$

for the first two terms $F_{0}$ and $F_{1}$ in the expansion for the stream function.

The solution to Eq. 11 gives

$$
F_{0}=\frac{4 \zeta^{2}}{64 / 3+\zeta^{2}}
$$

while the solution to Eq. 12 takes the form

$$
F_{1}=-x_{o}\left(F_{0}-\zeta F_{0}^{\prime}\right),
$$

where $x_{\mathrm{o}}$ is a constant to be determined below. Substituting these expressions into $\mathrm{Eq} .10$ gives $\psi=x F_{0}-x_{0}\left(F_{0}-\zeta F_{0}^{\prime}\right)+O\left(x^{-1}\right)$, which can be written at the same level of approximation as

$\psi=\left(x-x_{o}\right) F_{0}\left(\frac{r}{x-x_{o}}\right)+O\left(x^{-1}\right)$.

indicating that the first correction to Eq. 13 represents both a translation of the axial coordinate and a dilatation of the radial coordinate, which are incorporated through the apparent origin $x_{\mathrm{o}}$. Writing the two-term expansion for $\psi$ in this form therefore demonstrates that the virtual origin is not an ad-hoc improvement that one needs to introduce to avoid the singular behavior of $u$ as $x \rightarrow 0$, but that it emerges naturally as the first correction to the leadingorder result for $x \gg 1$.

The value $x_{o}$, which cannot be arbitrarily selected, must be computed from continuity considerations [15] by evaluating Eq. 9 in the far-field region. At distances $x \gg 1$, the volume flux driven by the jet can be calculated aside from a factor $2 \pi$ as $\int_{0}^{\infty} r u d r=F_{0}(\infty)\left(x-x_{0}\right)=$ $4\left(x-x_{0}\right)$ and the entrainment rate approaches the asymptotic far-field value $\chi=\left(F_{0}-\zeta F_{0}^{\prime}\right)$ $(\infty)=4$. Introducing these expressions into $\mathrm{Eq}$. 9 finally yields

$x_{o}=-\left[\int_{0}^{\infty}(\chi / 4-1) \mathrm{d} x+g_{o} / 8\right]$,

where the variable entrainment rate $\chi(x)$ must be determined by integration of Eqs. 1 and 2 . The location $x_{\mathrm{o}}$ of the virtual origin depends therefore on the initial velocity distribution $\bar{u}(r)$ through the value of $g_{o}$ and also through the entrainment-rate distribution $\chi(x)$. Sample values of $x_{\mathrm{o}}$ obtained for a uniform profile and for a parabolic profile are, respectively $x_{\mathrm{o}}=-0.230$ and $x_{\mathrm{o}}=-0.168$. As expected, these exact values differ moderately from those determined previously in the approximate calculations of Rankin and Sridhar [8] and Lee et al. [9]. Their predictions for the virtual origin can be written in terms of the axial coordinate used here as 
$x_{\mathrm{o}}=-0.254$ for the uniform velocity profile [8] and $x_{0}=-0.108$ for the parabolic velocity profile [9].

The description of the mass fraction distributions in the far field also makes use of expansions in inverse powers of $x$. Because the solution for $\left(1-\hat{Y}_{o}\right)$ follows from that of $\hat{Y}_{F}$ by replacing the Schmidt number of the fuel with that of the oxidizer, we restrict the presentation here to the development for $\hat{Y}_{F}$. Introducing the expansion

$\hat{Y}_{F}=x^{-1} Y_{0}(\zeta)+x^{-2} Y_{1}(\zeta)+O\left(x^{-3}\right)$

into 3,6 , and 7 yields the leading-order problem

$\left(\zeta Y_{0}^{\prime}+S_{F} F_{0} Y_{0}\right)^{\prime}=0 ; \quad\left\{\begin{array}{l}Y_{0}^{\prime}(0)=Y_{0}(\infty)=0 \\ 2 \int_{0}^{\infty} F_{0}^{\prime} Y_{0} \mathrm{~d} \zeta=g_{o}\end{array}\right.$

which can be solved to give

$Y_{0}=\frac{\left(2 S_{F}+1\right) g_{o} / 8}{\left(1+3 \zeta^{2} / 64\right)^{2 S_{F}}}$

On the other hand, the first-order correction $Y_{1}$ is obtained from

$$
\begin{aligned}
Y_{1}^{\prime \prime} & +Y_{1}^{\prime}\left(1+S_{F} F_{0}\right) / \zeta \\
& +Y_{1}\left(2 S_{F} F_{0}^{\prime}\right) / \zeta=-x_{0} S_{F} Y_{0} F_{0}^{\prime \prime} ; \\
& \left\{\begin{array}{l}
Y_{1}^{\prime}(0)=Y_{1}(\infty)=0 \\
\int_{0}^{\infty}\left(F_{0}^{\prime} Y_{1}+F_{1}^{\prime} Y_{0}\right) \mathrm{d} \zeta=0
\end{array}\right.
\end{aligned}
$$

This problem admits the particular solution

$Y_{1}=x_{0}\left(Y_{0}+\zeta Y_{0}^{\prime}\right)$

where $x_{0}$ is the virtual origin of the velocity distribution given in Eq. 16. The particular solution 21 satisfies the homogeneous boundary conditions as well as the additional integral constraint $\int_{0}^{\infty}\left(F_{0}^{\prime} Y_{1}+F_{1}^{\prime} Y_{0}\right) \mathrm{d} \zeta=0$ arising from 7. Because the linear homogeneous problem associated with 20 possesses no solution other than the trivial one $Y_{1}=0$, we may conclude that 20 possesses a unique solution given by 21 .

Combining now the first two orders in the expansion for $\hat{Y}_{F}$, given in Eqs. 19 and 21, yields
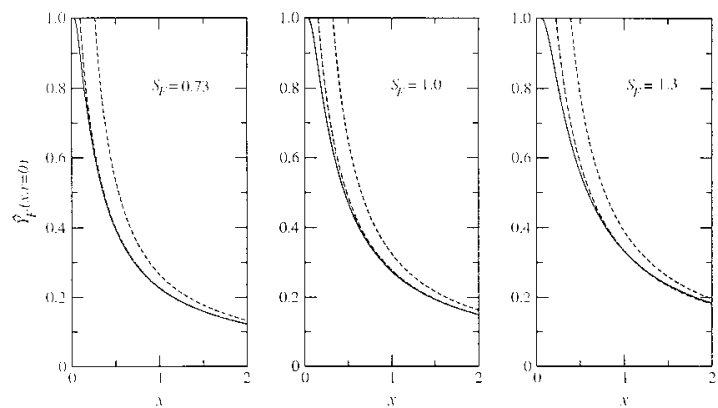

Figure 4. The variation of $Y_{F}$ along the axis as obtained from numerical integration of 1-6 (solid lines), from the leading-order far-field result $Y_{F}=g_{o}\left(2 S_{F}+1\right) /(8 x)$ (dashed lines), and from the corrected distribution $Y_{F}=g_{o}\left(2 S_{F}+\right.$ $1) /\left[8\left(x-x_{o}\right)\right]$ (long dashed lines).

$\hat{Y}_{F}=\frac{1}{x-x_{0}} Y_{0}\left(\frac{r}{x-x_{0}}\right)+O\left(x^{-3}\right)$

for the fuel mass fraction, indicating that the virtual origin of the velocity field also enters as the first correction to the the reactant distribution. In summary, the above results give with relative errors of order $S^{-2}$

$$
\begin{aligned}
\frac{8}{3}\left(x-x_{o}\right) u & =\left(\frac{8\left(x-x_{o}\right) \hat{Y}_{F}}{g_{o}\left(2 S_{F}+1\right)}\right)^{1 / S_{F}} \\
& =\left(\frac{8\left(x-x_{o}\right)\left(1-\hat{Y}_{o}\right)}{g_{o}\left(2 S_{o}+1\right)}\right)^{1 / S_{o}} \\
& =\left[1+\frac{3}{64}\left(\frac{r}{x-x_{o}}\right)^{2}\right]^{-2}
\end{aligned}
$$

for the solution to $1-4$ at distances $x \sim S \gg 1$.

It is remarkable that the virtual origin of the composition field is equal to that of the velocity field, and therefore independent of the Schmidt number. This surprising behavior can be explained by noting that it is the first-order correction to the velocity field that produces the driving term arising on the right-hand side of Eq. 20, which in turn forces a translation of the axial coordinate and a dilatation of the radial coordinate of magnitude $x_{\mathrm{o}}$ for $\hat{Y}_{F}$. Because the first-order correction to the composition field is due to the modified velocity field, the resulting virtual origin is independent of molecular diffusion. This result is tested in Fig. 4 where the variation of $\hat{Y}_{F}$ along the axis obtained from numerical integration of 1-6 is compared with the leading-order result $\hat{Y}_{F}=g_{o}\left(2 S_{F}+1\right) /(8 x)$ 
and also with the corrected distribution $\hat{Y}_{F}=$ $g_{o}\left(2 S_{F}+1\right) /\left[8\left(x-x_{o}\right)\right]$. As can be seen, the fuel distribution corrected with the virtual origin $x_{0}$ $=-0.168$ reproduces accurately the numerical results for the three values of $S_{F}$ considered in the plot.

The description 23 characterizes frozen mixing in the jet, and it is therefore applicable together with the computation methodology of Chung to calculate lift-off heights. The distribution of equivalence ratio $\phi=S \hat{Y}_{F} / \hat{Y}_{O}$ can be easily computed from 23 to give

$$
\begin{aligned}
\phi= & \left(\frac{1}{X} \frac{1}{\left(1+R^{2} / X^{2}\right)^{2 S_{F}}}\right) \\
& \cdot\left[1-\frac{1}{S} \frac{1}{X} \frac{2 S_{O}+1}{2 S_{F}+1} \frac{1}{\left(1+R^{2} / X^{2}\right)^{2 S_{O}}}\right]^{-1},
\end{aligned}
$$

where

$X=\frac{8\left(x-x_{o}\right)}{g_{o}\left(2 S_{F}+1\right) S}$ and $R=\frac{\sqrt{3} r}{g_{o}\left(2 S_{F}+1\right) S}$

are appropriately rescaled coordinates, of order unity in this far-field region.

Equating (24) to unity determines the axial variation of the radius of the stoichiometric surface, which is given at leading order by

$R_{s}=X\left(X^{-1 /\left(2 S_{F}\right)}-1\right)^{1 / 2}$.

As can be seen, the radius decreases with distance if $S_{F} \leqslant 1 / 4$ and reaches a maximum value given approximately by $R_{\max } \simeq X_{\max } \sim \sqrt{4 S_{F}-1}$ at an intermediate location $X_{\max } \simeq\left[\left(4 S_{F}-\right.\right.$ 1) $\left./\left(4 S_{F}\right)\right]^{2 S_{F}}$ when $S_{F}>1 / 4$. Notice that $X=1$ gives, in the first approximation, the downstream end of the stoichiometric surface. Using this result in the first equation of 25 yields at leading order $\left[g_{o}\left(2 S_{F}+1\right) / 8\right] S \operatorname{Re} a \simeq 0.3 \mathrm{~S} \operatorname{Re}$ $a$ for the maximum lift-off height. As expected, the far-field description only applies for $S \gg 1$; for $S$ of order unity, the lift-off height resulting from Schlichting solution is of order $0.3 \operatorname{Re} a$, corresponding to a location within the jet development region where the far-field solution is no longer valid.

Retaining smaller terms of order $S^{-1}$ in the expression for $R_{S}$ yields

$$
\begin{aligned}
R_{s}= & X\left\{X^{-1 /\left(2 S_{F}\right)}\right. \\
& \left.\cdot\left[1+\frac{1}{2} \frac{1}{S} \frac{1}{S_{F}} \frac{2 S_{O}+1}{2 S_{F}+1} X^{S_{O} / S_{F}-1}\right]-1\right\}^{1 / 2} .
\end{aligned}
$$

On the other hand, the flow velocity along the stoichiometric surface is given with relative errors of order $S^{-1}$ by

$u_{s}=\frac{3}{g_{o}\left(2 S_{F}+1\right) S} X^{1 / S_{F}-1}$

and with relative errors of order $S^{-2}$ by

$$
\begin{aligned}
u_{s}= & \frac{3}{g_{o}\left(2 S_{F}+1\right) S} X^{1 / S_{F}-1} \\
& \cdot\left(1-\frac{1}{S} \frac{1}{S_{F}} \frac{2 S_{O}+1}{2 S_{F}+1} X^{S_{o} / S_{F}-1}\right) .
\end{aligned}
$$

Combining Chung's equation $u_{s}=U_{f} / U_{o}$ with the above distributions of $u_{s}$ determines the lift-off height in lifted flames with $S \gg 1$. For instance, use of the leading-order distribution 28 gives

$X_{f}=\left(\frac{g_{o}\left(2 S_{F}+1\right)}{3} \frac{S U_{f}}{U_{o}}\right)^{S_{F} /\left(1-S_{F}\right)}$

for the lift-off height normalized with the length of the stoichiometric surface. Clearly, because the stoichiometric surface ends at $X=1$, for the location to represent a flame front stabilized at the stoichiometric surface the value of $X_{f}$ obtained from the above equation must be no larger than unity, with the condition $X_{f}=1$ representing critical conditions for blowoff. As previously explained, an additional condition for the stability of the resulting flame in this far-field region is $S_{F}>1$.

The accuracy of 26-29 is tested in Figs. 1 and 2. For instance, the shape of the stoichiometric surface obtained numerically for $S=2,4,8,16$ is compared in Fig. 1 with the two-term asymptotic prediction 27. As can be seen, excellent agreement is found in these cases of moderately large $S$. The simpler leading-order prediction 26 also gives reasonable accuracy for the case $S=$ 16 considered. Similarly, the two-term prediction 29 for $u_{s}$ is compared in Fig. 2 with the 
boundary-layer solution. As expected, relatively large errors are found in the developing region $x \sim 1$, where Schlichting distribution is not applicable. Further downstream, the agreement is excellent. The comparison of the leadingorder prediction 28 in the case $S_{F}=1.3$ reveals that the first-order correction is necessary for increased accuracy.

To further illustrate the inadequacy of Schlichting solution to describe mixing when $S \sim 1$, Eq. 28 is used in Fig. 3 to evaluate $u_{s}$ for $S=1$ and different values of $S_{F}$. As previously anticipated, departures of order unity appear everywhere. Moreover, the far-field solution incorrectly yields a decrease of $u_{s}$ with distance when $S_{F}=1.3$, and therefore, leads to incorrect predictions concerning the stability of lifted flames with dilute fuel feed. It is worth noting that no improvement follows in this case from using the first-order correction, that is, the use of Eq. 29 for $S=1$ leads to negative values of $u_{s}$.

\section{LIFTED FLAMES IN AXISYMMETRIC FUEL JETS}

Both curvature effects and thermal expansion effects may affect the propagation velocity $U_{f}$ of the triple flame travelling against the fuel jet. For instance, analyses incorporating a constantdensity assumption [4, 5] have demonstrated that flame-front curvature decreases the value of $U_{f}$ from the propagation velocity of a stoichiometric premixed flame. $S_{L}$, when the radius of curvature of the triple flame becomes comparable to the front thickness. We shall see below that this effect is unimportant in the configuration studied here, for which the Reynolds number is sufficiently larger than unity.

To estimate the radius of curvature of the premixed fronts appearing in round jets, one may use the approximate law

$U_{L} / U_{m}=1-B\left(\phi-\phi_{m}\right)^{2}$,

for the dependence of the propagation velocity of a planar premixed flame $U_{L}$ on the mixture composition, where the heuristic constants $B$ and $\phi_{m}$ take different values for different fuels [16]. The equation above shows a equivalenceratio dependence of $U_{L}$ somewhat more general than that postulated in Chung's work, in that it is assumed that the peak value of $U_{L}=U_{m} \neq S_{L}$ is achieved at $\phi=\phi_{m}$ and not precisely at $\phi=$ 1. Because $\phi_{m} \simeq 1$ for most hydrocarbon-air flames, for example, $\phi_{m}=1.08$ for propane and $\phi_{m}=1.13$ for isooctane [16], this difference is not very significant and $U_{m} \simeq \mathrm{S}_{\mathbf{L}}$ for most fuels. On the other hand, the constant $B$ is moderately large in most cases, taking for instance the values $B=4.05$ for propane and $B=3.22$ for isooctane [16].

For lifted flames in round jets, the variation of $U_{L}$ with $\phi$ given above in Eq. 31 indicates that the radius of curvature of the flame front is a factor $B$ smaller than the local jet radius. This radius of curvature is to be compared with the flame front thickness, which can be estimated to be of order $v / S_{L}$. Different scalings appear for $S$ $\sim 1$ and for $S \gg 1$. Thus, if $S \sim 1$, the flame would be stabilized at a lift-off height of order $\operatorname{Re} a$, in a region where the value of $U_{f}$ equates the flow velocity, of order of the initial jet velocity $U_{o}$, and where the local radius is of order $a$. On the other hand, if $S \gg 1$, then the flame would be stabilized farther from the injector, at distances of order $S$ Re a where the flow velocity and the local radius are of order $U_{o} / S$ and $S a$, respectively. Correspondingly, characteristic values for the thickness and for the radius of curvature of the flame front are $v / U_{o}$ and $a / B$ if $S \sim 1$ and $S v / U_{o}$ and $S a / B$ if $S \gg$ 1. Although the scalings are different, we may conclude in both cases that curvature effects will be negligible as long as $R e \gg B$, a criterion satisfied in most practical applications. The local structure of the flame front is therefore that of a planar premixed front, with a lean branch that extends outwards to a radius where the mixture reaches its lean flammability limit and with a rich branch that ends at an inner radius with $\phi$ equal to the rich flammability limit, $\phi_{r}$ or would reach the axis if the equivalence ratio there, $\phi=\phi_{a}$, were smaller than $\phi_{r}$.

Thermal expansion effects influence the value of $U_{f}$ [6]. The flow in the nose region downstream from the flame is rotational, with overpressures that deflect the incoming streamlines outwards, and slow the flow velocity along $\Sigma_{s}$. Correspondingly, the front propagation velocity relative to the unperturbed flow is somewhat larger than $S_{L}$. This effect was numerically 
ascertained by Ruetsch et al. [6] for a symmetric $(S=1)$ two-dimensional triple flame propagating against a stream with an initially uniform velocity profile. Calculations of propagation velocities with $S \neq 1$, including thermal expansion effects and nonuniform velocity profiles, are a suitable subject of future work.

The propagation of triple flames in round jets when $S \gg 1$ should also be addressed in future research. The above scales suggest that, for a typical hydrocarbon-air flame with $B \geq 1$, the flame front exhibits a radius of curvature comparable to the local jet radius, of order $S a$ and therefore extends across the fuel jet, giving an inherently axisymmetric structure that has been observed for instance in the experiments reported in [17]. Calculations of such flames should employ the distributions of velocity and composition given in 23 as an appropriate boundary condition upstream from the flame.

Although thermal expansion effects are anticipated to affect significantly the motion of these axisymmetric fronts by a mechanism analogous to that affecting the propagation of two-dimensional triple flames [6], we shall use here a constant-density model to illustrate the effect of the radial velocity gradient on the flame shape. In this thermal-diffusive approximation, the structure of a triple flame stabilized at an axial location $X=X_{f}$ is determined simply by a local kinematic balance between the front propagation velocity $U_{L}$ and the normal component of the local flow velocity $U=U_{o} u$ to give

$\epsilon= \pm \int_{\eta t}^{\eta} \frac{\sqrt{1-\left(U_{L} / U\right)^{2}}}{U_{L} / U} d \eta$

for the flame shape. Here, the radial coordinate $\eta$ and the axial distance to the leading edge of the flame, $\xi$, are both scaled with the same distance $\left[\mathrm{g}_{\mathrm{o}}\left(2 S_{F}+1\right) / \sqrt{3}\right]\left(X_{f} S a\right)$, which is the characteristic value of the local jet radius at $X=X_{f}$ a quantity of order $S a$. The leading edge of the flame is located at a radial location $\eta_{1}$ where the conditions $U=\mathrm{U}_{\mathrm{L}}$ and $\mathrm{d}\left(U-U_{L}\right) / \mathrm{d} \eta=0$ are simultaneously satisfied. The plus sign in front of the above integral corresponds to the lean branch $(\eta>$ $\eta_{l}$ ), while the minus sign should be used for the rich branch $\left(\eta<\eta_{l}\right)$.

The calculation of triple flames from Eq. 32 necessitates additional information regarding the dependence of $U_{L}$ on the local mixture composition. Curves $U_{L}(\phi)$ of the type needed here have been compiled for instance by Law [18]. Combining this information with the distribution of equivalence ratio given in Eq. 23 provides the function $U_{L}(\eta)$ to be introduced into Eq. 32.

The computations presented below make use of the parabolic representation Eq. 31, which, with appropriate choices for $U_{m}, \phi_{m}$, and $B$, approximates accurately the curves $U_{L}(\phi)$ over a wide range of values of $\phi$ about $\phi_{m}$. Note that, because 31 predicts a zero propagation velocity at $\phi=\phi_{m} \pm B^{-1 / 2}$, the resulting flame fronts extend for $\phi$ between the limiting values $\phi=$ $\phi_{m} \pm B^{-1 / 2}$, which thereby act effectively as flammability limits in determining the transverse extent of the flame. Using 31 together with 23 yields

$$
\begin{aligned}
\phi_{a}\left(1+\eta_{l}^{2}\right)^{-2 S_{F}=} & \phi_{m}\left[1-\frac{S_{F}}{2 S_{F}-1}\right. \\
& \left.\cdot\left(1-\sqrt{1-\frac{2 S_{F}-1}{B S_{F}^{2} \phi_{m}^{2}}}\right)\right],
\end{aligned}
$$

and

$$
\begin{aligned}
\frac{U_{L}}{U}=\frac{1-B\left[\phi_{a}\left(1+\eta^{2}\right)^{-2 S_{F}}-\phi_{m}\right]^{2}}{1-B\left[\phi_{a}\left(1+\eta_{l}^{2}\right)^{-2 S_{F}}-\phi_{m}\right]^{2}} \\
\cdot\left(\frac{1+\eta^{2}}{1+\eta_{l}^{2}}\right)^{2}
\end{aligned}
$$

for the radial location of the leading edge of the flame, $\eta_{l}$, and for the velocity ratio $U_{L} / U$ to be introduced into 32. Equation 33, which can be solved for $\eta_{l}$ provided $B>\left(2 S_{F}-1\right) /\left(S_{F} \phi_{m}\right)^{2}$, is written for the local equivalence ratio at the leading edge, $\phi_{l}=\phi_{a}\left(1+n_{l}^{2}\right)^{-2 S_{F}}<\phi_{m}$, with $\phi_{a}=X_{f}^{-1}$ denoting the value of the equivalence ratio at the axis.

Integration of Eq. 32 supplemented with Eq. 33 and Eq. 34 provides the flame shape. For a given fuel (with given chemical-kinetic parameters $\phi_{m}$ and $B$ and a given Schmidt number $S_{F}$ ), the solution for the flame front can be charac- 


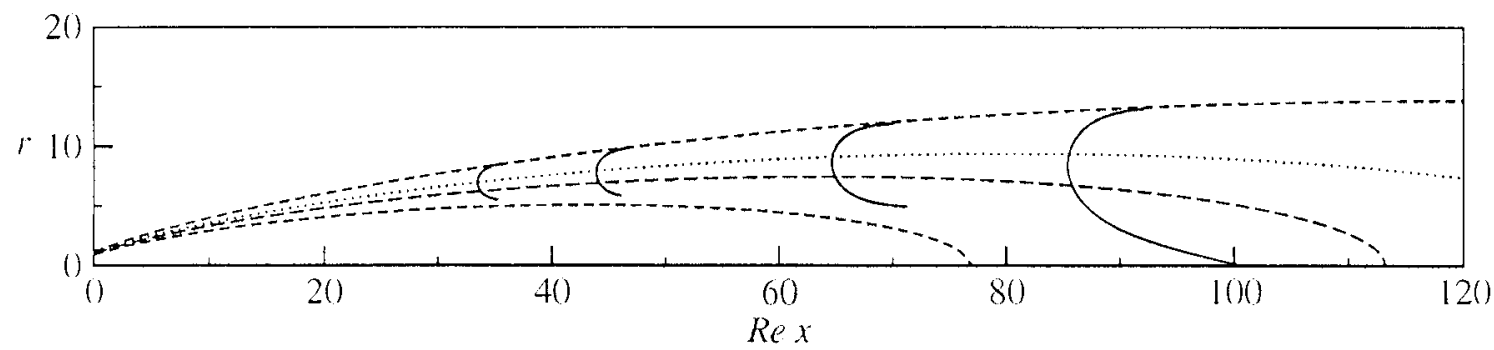

Figure 5. Flame fronts obtained from 32-34 with $\phi_{m}=1.08, B=4.05, S_{F}=1.3$, and $X_{j}=(0.25,0.33,0.50,0.67)\left[\phi_{a}=(4.0\right.$, $3.0,2.0,1.5)]$. The plot exhibits the flame fronts superposed on a fuel jet with $R e=20$ and $S=16$, with the composition field being characterized by the isosurfaces $\phi=\phi_{m}$ (long dashes), $\phi=\phi_{i}$ (dots) and $\phi=\phi_{m} \pm B^{-1 / 2}$ (dashes).

terized in terms of the normalized lift-off height $X_{f}$, which can be computed in the first approximation from 30 for a given value of $S U_{l} / U_{o} \simeq S$ $S_{I} / U_{\mathrm{o}}$. Characteristic flame shapes corresponding to propane $\left(\phi_{m}=1.08 . B=4.05\right.$, and $S_{F}=$ 1.3) are exhibited in Fig. 5 for $X_{f}=(0.25,0.33$, $0.50,0.67)$. The plot shows the corresponding flame fronts as they would appear downstream from a fuel injector with $R e=20$ and $S=16$, with the composition field being characterized in the plot by the iso-surfaces $\phi=\phi_{\mathrm{m}}=1.08$, $\phi=\phi_{l}$ and $\phi=\phi_{m} \pm B^{-1 / 2}$. To represent realistically the slenderness of the solution, both the radius and the axial distance to the injector are scaled here with the injector radius.

As expected, the flames located farther downstream are wider, and eventually extend all the way across the jet for sufficiently small values of $\phi_{a}=X_{f}^{-1}<\phi_{m}+B^{-1 / 2}$. Also, since $\phi_{l}$ is always smaller than $\phi_{m}$, the leading edge is seen to be slightly displaced outwards from the stoichiometric surface, an effect of the nonuniform velocity profile that becomes more noticeable for flames lying farther from the injector. The displacement observed would be less pronounced for larger values of $B$, as can be anticipated by writing 33 for $B \gg\left(2 S_{F}-1\right) /\left(S_{F} \phi_{m}\right)^{2}$ in the form $\phi_{l}=\phi_{m}$ $\left[1-1 /\left(2 B S_{F} \phi_{m}^{2}\right)\right]$.

Similar trends are expected to emerge when variable density is accounted for. In that case, the solution not only depends on the normalized lift-off height $X_{f}$ but also on an additional heat-release parameter measuring the exothermicity of the reaction [6]. The required computational approach should be analogous to that employed in [6], although in this axisymmetric case the computational difficulties associated with streamline deflection far from the flame are expected to be less stringent than those encountered in the previous two-dimensional calculations.

Clearly, numerous problems concerning triple flames stabilized in laminar fuel jets remain to be solved. The analysis given above can help the needed investigations by providing an accurate description for the mixing process of the fuel jet with the surrounding air in terms of the minimum number of parameters. We have seen that, if $S \sim 1$, then integration of Eqs. $1-6$ is required to determine the distribution of flow velocity and mixture composition upstream from the premixed front, while the far-field solution 23 provides the needed description when $S \gg 1$. Extension of the above boundarylayer analysis to include effects of coaxial air flow and outer confinement on the mixing process is anticipated. As previously discussed, for the calculation of lift-off heights, the present results must be supplemented with careful evaluations of propagation velocities $U_{f}$ including both thermal expansion effects, as in [6], and also geometrical effects associated with the axisymmetric structure of lifted flames when $S \gg$ 1. These evaluations, not currently available, constitute a worthwhile topic for future research.

The work of $A R$ and $A L S$ was supported by the Spanish DGESIC under contract number PB980142-C04-02, while that of $A L$ was supported by the Spanish CICYT under contract no PB940400 .

\section{REFERENCES}

1. Chung, S. H., and Lee, B. J., Combust. Flame 86:62 (1991) 
2. Lee, B. J., and Chung, S. H., Combust. Flame 109:163 (1997).

3. Won, S. H., Chung, S. H., Cha, M. S., and Lee, B. J. "Lifted flame stabilization in developing and developed regions of coflow jets for highly diluted propane," Proceedings of the Combustion Institute 28:2093-2099 (2000).

4. Dold, J. W., Combust. Flame 76:71 (1989).

5. Hartley, L. J., and Dold, J. W., Combust. Sci. Tech. 80:23 (1991).

6. Ruetsch, G. R., Vervish, L., and Liñán, A. Phys. Fluids $A$ 7:1447 (1995).

7. Chen, Y. C., and Bilger R. W. Combust. Flame 123:23 (2000).

8. Rankin, G. W., and Sridhar, K. J. Fluids Eng. 100:55 (1978).

9. Lee, D. S., Kihm, K. D., and Chung, S. H. J. Fluids Eng. 119:716 (1997).

10. Schlichting, H. Z. Angev. Math. Mech. 13:260 (1933).

11. Squire, L. C. Quart. J. Mech. 4:321 (1951).
12. Ghosal, S., and Vervisch, L., Combust. Flame 124:646 (2001).

13. Ko, Y. S., and Chung, S. H., Combust. Flame 118:151 (1999).

14. Ko, Y. S., Chung, S. H., Kim, G. S., and Kim, S. W. Combust. Flame 123:430 (2000).

15. Revuelta, A., Sánchez, A. L., and Liñán, A. "Confined axisymmmetric laminar jets with large expansion ratios," J. Fluid Mech. (accepted for publication, 2001).

16. Metghalchi, M., and Keck, J. C., Combust. Flame 48:191 (1982).

17. Plessing, T., Terhoeven, P., Mansour, M. S., and Peters, N., Combust. Flame 115:335 (1998).

18. Law, C. K., in Reduced Kinetic Mechanisms for Applications in Combustion Systems (N. Peters and B. Rogg, Eds.) Springer Verlag, New York, 1993, pp. 15-26.

Received 28 April 2001; revised 22 September 2001; accepted 22 October 2001 